\title{
PROYECTO ACRA: EXPERIENCIAS DIDÁCTICAS EN CIENCIAS CON REALIDAD AUMENTADA EN LOS NIVELES PRE-UNIVERSITARIOS
}

\section{ACRA PROJECT: DIDACTIC EXPERIENCES IN SCIENCE WITH AUGMENTED REALITY AT PRE-UNIVERSITY LEVELS}

\author{
Lourdes Villalustre Martínez; villalustrelourdes@uniovi.es \\ M.Esther Del Moral Pérez; emoral@uniovi.es \\ M.Rosario Neira Piñeiro; neiramaria@uniovi.es \\ Monica Herrero Vazquez; herreromonica@uniovi.es
}

Universidad de Oviedo

\section{RESUMEN}

Se describen 21 experiencias derivadas del Proyecto de Innovación ACRA (2016-17), impulsado por la Consejería de Educación asturiana e implementado en aulas de Infantil, Primaria, Secundaria y Bachillerato de 9 centros escolares-, que promueven la integración de la Realidad Aumentada (RA) en el proceso de enseñanza-aprendizaje de ciencias. Tras el análisis de contenido de las experiencias desarrolladas, se constata que sus objetivos se orientan a favorecer la comprensión de conceptos o procesos científicos y a explicar realidades o hechos ligados a la ciencia, adoptando metodologías activas, como el trabajo por proyectos. En conclusión, ACRA ha contribuido a explotar didácticamente la RA, ofreciendo una muestra de buenas prácticas para comprender mejor sus potencialidades ligadas a facilitar el aprendizaje de conceptos científicos.

PALABRAS CLAVE: realidad aumentada, competencia científica, educación pre-universitaria

\begin{abstract}
This work describes 21 experiences derived from the ACRA Innovation Project (2016-17) (Spanish acronym for Learning Sciences with Augmented Reality) promoted by the Asturian Department of Education, implemented at pre-university levels (kindergarten, elementary, middle and high school) in 9 formal educational institutions. The project looked for the integration of Augmented Reality (RA) in the teaching-learning processes of natural sciences. Experiences analyzed revealed that their objectives were oriented to facilitate the understanding of scientific concepts or processes and to explain facts and natural phenomena, adopting active methodologies such as project work. In conclusion, ACRA project has contributed to spread didactically the use of $R A$, selecting a sample of good practices to better understand its potential for improving learning of scientific concepts.
\end{abstract}

KEYWORDS: augmented reality, scientific competence, pre-university education. 


\section{INTRODUCCIÓN}

La irrupción tanto de los dispositivos móviles como de las nuevas aplicaciones tecnológicas en las aulas, tales como la realidad aumentada (RA), está favoreciendo la emergencia de experiencias didácticas innovadoras -especialmente en la enseñanza de las ciencias- en los contextos educativos no universitarios (Leiva y Moreno, 2015; Sánchez-Prieto, Olmos, García-Peñalvo y Torrecilla, 2016; Villalustre y Del Moral, 2016a), por entender que pueden facilitar la adquisición de la competencia científica y habilidades de distinta índole en el alumnado (De la Torre, Martín-Dorta, Saorín, Carbonell y Contero, 2015; Solano, Díaz, Casas y Guevara, 2015), mejorar sus capacidades cognitivas (Del Cerro y Morales, 2017), así como incrementar su motivación por temáticas ligadas a las ciencias naturales (Toledo y SánchezGarcía, 2017).

Dada la variedad de definiciones, taxonomías y categorías de la RA, se ha propuesto recientemente la visión de la RA como un concepto más que como un tipo de tecnología, lo que podría ser más efectivo para educadores, investigadores y diseñadores (Wu, Lee, Chang y Liang, 2013).

Evidentemente, la RA -en sus distintos niveles de aplicación- ofrece fórmulas atractivas que acercan a la realidad de un modo diferente, permitiendo visibilizar procesos complejos, que bien por su grado de abstracción, naturaleza científica o espacial no son fáciles de aprehender, es decir, asimilar o comprender en su totalidad (Cubillo, Martín-Gutiérrez, Castro y Colmenar, 2014; Del Moral y Villalustre, 2013). Asimismo, proporciona experiencias de aprendizaje ligadas a la exploración y descubrimiento de información complementaria conectada con el mundo real, a través de códigos QR u otro tipo de elementos activadores como marcadores, que están aprovechando en ámbitos como el turístico o museístico (Caro, Luque y Zayas, 2014). De modo semejante, la geolocalización permite combinar actividades formativas dentro y fuera del aula, logrando aproximar la realidad y el medio natural al alumnado, garantizando así que su aprendizaje sea realmente significativo y situado (Villalustre y Del Moral, 2016b).

Es indudable que la escuela debe asumir los cambios impuestos por la emergencia de estas nuevas tecnologías (Méndez, 2012), y que el profesorado debe interiorizarlos para mejorar su práctica docente (Sancho, Correa, Giró, y Fraga, 2014). Ello requiere optar por metodologías alternativas que contemplen -entre otras- la RA como una aliada indiscutible que contribuya a optimizar el proceso de enseñanza-aprendizaje -en este caso, de las ciencias-, incrementando la comprensión de determinados fenómenos científicos y naturales, haciéndolos más tangibles (De Pedro y Martínez-Méndez, 2012) al utilizar modelos 3D, e incluso, dotándolos de la cuarta dimensión (Rosado, 2010) a partir de las notaciones sociales con las que los aprendices y usuarios van enriqueciendo la realidad.

Sin embargo, para que esto sea posible, la clave se halla en la necesaria cualificación y formación específica del profesorado en donde se cualifique no solo el manejo de los dispositivos móviles o tecnologías digitales, sino también se les dote de estrategias didácticas necesarias para incorporar actividades formativas atractivas en las aulas apoyadas en la RA (Cabero, 2010), que busquen el logro de objetivos educativos concretos, y que 
favorezcan la resolución de problemas en los nuevos entornos tecnológicos y digitales (Salinas, 2012).

Además, las numerosas aplicaciones (app) y desarrollos tecnológicos apoyados en la RA que están surgiendo, tanto de libre acceso como comerciales, constituyen un banco de recursos de gran valor didáctico que los docentes deben conocer y explorar para valorar cómo pueden integrarlos en el currículum. En concreto, se han hecho algunas recopilaciones de apps sobre temáticas relacionados con el área de ciencias que pueden dar alguna pista para abordar contenidos científicos en Educación Infantil, Primaria y Secundaria Obligatoria (Villalustre y Del Moral, 2017).

Por otro lado, y si bien se observa que han proliferado prácticas innovadoras con RA en los distintos niveles educativos y áreas curriculares, aquí se pone el foco de atención en aquellas implementadas en el área de ciencias, principalmente en ciencias naturales -aunque también, en menor medida, en ciencias sociales-, tanto en Infantil y Primaria como en Secundaria y Bachillerato. Se trata de experiencias surgidas dentro del Proyecto de Innovación ACRA (2016-17), impulsado por la Consejería de Educación asturiana.

\section{POTENCIALIDAD DE LA RA EN LA ENSEÑANZA DE LAS CIENCIAS EXPERIMENTALES}

Tradicionalmente, la enseñanza de las ciencias experimentales adolece de una falta de sintonía entre sus planteamientos teóricos y los materiales curriculares que se emplean en las aulas (Martí, 2012). Esto se plasma en el informe ENCIENDE, publicado por la Confederación de las Sociedades Científicas de España (COSCE, 2011), donde se destaca la necesidad de implementar recursos innovadores en la formación del profesorado de ciencias, generar buenas prácticas docentes, así como promover la creación de materiales didácticos novedosos.

La concreción de la competencia científica propuesta en el informe "Taking science to school, learning and teaching science in grades K-8" (National Research Council, 2007) implica el desarrollo de cuatro grandes habilidades: conocer, usar e interpretar las explicaciones científicas sobre los sistemas naturales, generar y evaluar evidencias y explicaciones científicas, comprender la naturaleza y el desarrollo del conocimiento científico y mantener una actitud continuada de interés hacia la ciencia y las novedades científicas. Es en este último aspecto, aunque no el único, es donde se puede enmarcar el uso de la RA en las aulas, más aún, teniendo presente que los materiales didácticos utilizados condicionan las metodologías a emplear. Así el uso de libros de texto en las clases de ciencias se relaciona con una metodología tradicional ya bastante cuestionada, centrada en el profesor y basada en la mera transmisión pasiva del conocimiento científico. De ahí que la formación y actualización docente sea clave para adoptar metodologías activas, apoyadas en recursos didácticos que pongan al alumnado en el centro del proceso formativo (Davis y Krajcik, 2005).

El Informe Rocard (Rocard et al., 2007) destaca la necesaria renovación y apuesta por buenas prácticas en la enseñanza de las ciencias, que ayuden a incrementar el interés y rendimiento de los escolares en esta disciplina. Reconoce que el origen de la pérdida de motivación del alumnado hacia la ciencia, que se agrava desde primaria a secundaria radica -en gran 
medida- en la forma en que se enseña en estos niveles educativos, demasiado abstracta y memorística, e identifica como causa fundamental la falta de confianza y dominio del conocimiento de la disciplina de los docentes, sobre todo en el nivel de primaria.

Estos antecedentes justifican la necesidad de incorporar nuevos recursos didácticos innovadores en la enseñanza de las ciencias, con objeto de desarrollar las habilidades de los estudiantes del siglo XXI (Binkley et al., 2010). Ello supone un reto para el profesorado que necesita adecuar su competencia digital y didáctica a los nuevos desarrollos tecnológicos (Wu, Lee, Chang y Liang, 2013; Roblizo, Sánchez-Pérez y Cózar, 2015), lo que únicamente podrá lograrse si se implica en actividades formativas periódicas y se integra en comunidades de práctica que le permitan actualizarse, contactando con otros docentes en activo con inquietudes similares y participando en proyectos colaborativos innovadores.

Recientemente se comparó por primera vez la utilidad de la RA frente a otros materiales didácticos adoptados en la enseñanza de las ciencias en primaria (Hung, Chen y Huang, 2017), en concreto, se comparó el desempeño de alumnos de quinto grado de dos escuelas primarias utilizando tres tipos diferentes de materiales didácticos (gráficos en 2D en un libro, objetos físicos en 3D y objetos virtuales en 3D en un libro de RA) para aprender el nombre de 6 bacterias diferentes en intervalos de tiempo. Los resultados mostraron que la RA del libro gráfico fue lo que más gustó a los alumnos, al considerarlo una fórmula práctica y activa para aprender, aunque su capacidad para recordar los contenidos fue similar tras utilizar los tres tipos de materiales.

Estos resultados están de acuerdo con las evidencias de Wu, Lee, Chang y Liang (2013), según los cuales, la RA puede facilitar: (1) contenidos de aprendizaje en perspectivas 3D, (2) el aprendizaje ubicuo, colaborativo y localizado, (3) la sensación de presencia, de inmediatez e inmersión, (4) la visualización de lo invisible y (5) la construcción de puentes entre el aprendizaje formal e informal. Estos aspectos pueden contribuir a la necesaria renovación del proceso de enseñanza-aprendizaje de las ciencias en las edades tempranas. Sin embargo, no todas estas ventajas son exclusivas de la RA ya que también son compartidas por el aprendizaje móvil o ubicuo (Wu, Lee, Chang y Liang, 2013).

Para aprovechar las posibilidades que brinda la RA es necesario explorar cómo implementarla adecuadamente en el aula para alcanzar los objetivos educativos propuestos (Bronack, 2011). Este aspecto es crucial para la enseñanza efectiva de las ciencias, superando la explotación reduccionista de la dimensión lúdica de la RA.

\section{PROYECTO ACRA: REALIDAD AUMENTADA PARA ABORDAR CONTENIDOS CIENTÍFICOS}

\subsection{Contextualización}

ACRA es un proyecto innovador centrado en la utilización didáctica de la RA para potenciar el desarrollo y la adquisición de contenidos científicos en el alumnado de educación infantil, primaria, secundaria y bachillerato. En el cual participaron 9 centros educativos ubicados en diferentes zonas geográficas de Asturias, y se planificaron y desarrollaron 21 experiencias. 
Previo a su desarrollo, el profesorado participante requirió formación específica sobre el manejo y aprovechamiento didáctico de este nuevo recurso, pues supuso para muchos su primer acercamiento a la RA, lo que condicionó en gran medida el desarrollo de las prácticas formativas llevadas a cabo. De igual modo, muchos de los profesores se constituyeron en grupos para desarrollar experiencias formativas conjuntas, aprovechando las sinergias del trabajo colaborativo.

\subsection{Objetivos}

El proyecto ACRA pretendía potenciar el aprendizaje y la adquisición de contenidos científicos -junto a otros de carácter digital-, en el alumnado de los niveles educativos no universitarios. Así, con él se quería:

- Promover el diseño y la reutilización de recursos digitales interactivos con RA entre el profesorado integrado en el proyecto, para favorecer el aprendizaje y la adquisición de contenidos científicos.

- Implementar prácticas formativas con creaciones diseñadas o reutilizables de RA, atendiendo a los distintos niveles de inmersión tecnológica tanto del profesorado como del alumnado, mediante geolocalización, creación de códigos $\mathrm{QR}$, animaciones 3D, etc.

- Analizar las diferentes prácticas formativas desarrolladas para la integración de la RA en los procesos de enseñanza-aprendizaje de ciencias en diferentes etapas educativas (Infantil, Primaria, Secundaria y Bachillerato).

\subsection{Fases del proyecto}

El proyecto fue coordinado por el equipo de investigación TECN@ de la Universidad de Oviedo, en el que participaron un total de 9 centros distribuidos por diferentes localidades del Principado de Asturias. El proyecto se articuló entorno a seis fases de desarrollo, como se expone en el gráfico 1: 


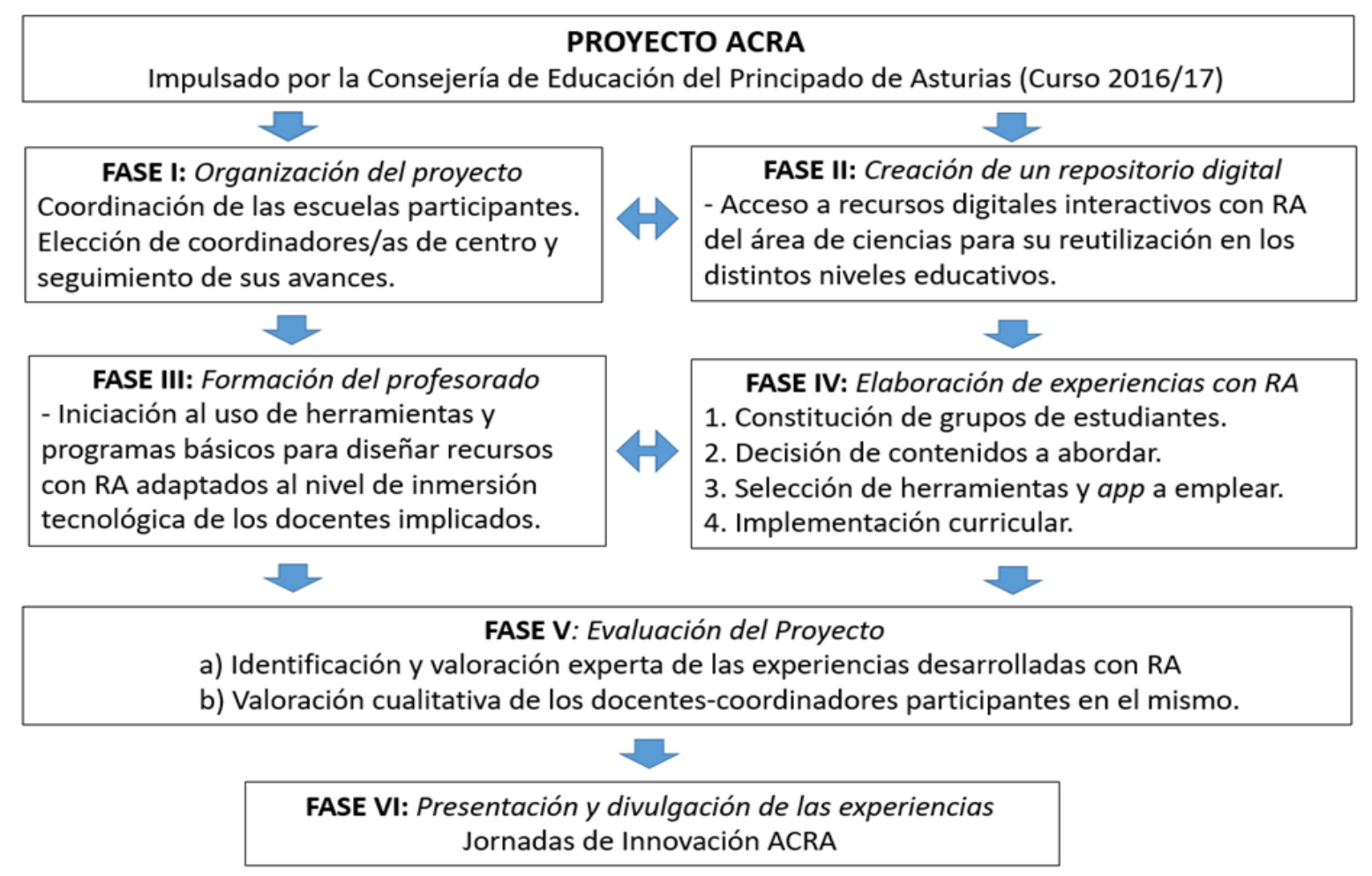

Gráfico 1. Fases implicadas en el desarrollo del proyecto ACRA

\subsection{Metodología}

Desde los diferentes centros implicados en el proyecto se planificaron y desarrollaron 21 experiencias formativas. Concluido éste, y tras solicitarles a los docentes que describieran sus experiencias, se adoptó una metodología basada en el análisis de contenido de las mismas (Villalustre, Del Moral, Neira-Piñeiro y Herrero, 2017), que siguió las pautas que se exponen:

\subsection{Pautas para el análisis de las experiencias}

El análisis de las experiencias formativas con RA para potenciar el desarrollo y adquisición de contenidos científicos, se efectuó analizando los siguientes aspectos:

1. Identificación de la experiencia: nombre dado por el profesorado a la experiencia desarrollada, y curso en el que se lleva a cabo.

2. Contenidos científicos abordados, según las categorías de Cañal (2012):

CCPC: Comprensión de Conceptos o Procesos Científicos

ERHC: Explicación de la Realidad o Hechos Científicos

DEPC: Diseño de Experimentos y Preguntas Científicas

IDPC: Interpretación de Datos y Pruebas Científicas

APGPC: Actitud positiva y gusto por el aprendizaje de la ciencia

3. Nivel de RA adoptado en la experiencia formativa, siguiendo la clasificación de Cawood y Fiala (2008): 
N1: Hiperenlaces en el mundo físico; mediante códigos QR.

N2: RA basada en marcadores; uso de marcadores para superponer objetos en 3D.

N3: RA markerless; utilización de imágenes y objetos como activadores.

N4: Visión aumentada; mediante el uso de las Google Glass o las lentillas biónicas.

4. Rol asumido por el docente: a) proveedor de recursos RA; b) facilitador del aprendizaje con RA; c) mediador de experiencias con RA.

5. Rol de los alumnos: a) consumidor activo de RA; b) explorador de recursos RA; c) creador de recursos RA.

6. Metodología adoptada: a) uso de la RA como apoyo a metodologías tradicionales; b) enfoques basados en proyectos, problemas o experimentación; c) uso de una variedad de enfoques.

\section{RESULTADOS}

Los centros participantes en el proyecto desarrollaron, desde diferentes perspectivas, actividades formativas, mostrando una gran variedad de enfoques y un despliegue de creatividad. Tras su desarrollo, y a partir de las pautas delimitadas se procedió al análisis de las experiencias diseñadas por el profesorado para abordar contenidos científicos mediante RA.

\subsection{Educación Infantil}

La RA proporciona un entorno óptimo para abordar de forma activa y consciente habilidades para la observación, la formulación y contraste de hipótesis y teorías, la elaboración de conclusiones, etc., siguiendo los principios que guían el pensamiento científico, de forma lúdica y entretenida. En este sentido, el profesorado implementó diferentes actividades, utilizando la RA para activar la curiosidad del alumnado por todo aquello que acontece a su alrededor y favorecer la comprensión y representación de la realidad, acercándoles a la misma y a sus relaciones causales (Tabla 1):

\begin{tabular}{|c|c|c|c|c|c|}
\hline $\begin{array}{l}\text { Contenidos } \\
\text { científicos }\end{array}$ & $\begin{array}{c}\text { Competencia } \\
\text { s científicas }\end{array}$ & Nivel de RA & Rol docente & Rol alumnos & Metodología \\
\hline \multicolumn{6}{|c|}{ Identificamos espacios (3으) } \\
\hline $\begin{array}{l}\text { Orientación } \\
\text { espacial, } \\
\text { puntos } \\
\text { cardinales }\end{array}$ & $\mathrm{CCPC}$ & $\begin{array}{l}\text { N1. } \\
\text { Mediante } \\
\text { códigos QR }\end{array}$ & $\begin{array}{l}\text { Facilitador } \\
\text { aprendizaje } \\
\text { con RA }\end{array}$ & $\begin{array}{l}\text { Explorador } \\
\text { de recursos } \\
\text { RA }\end{array}$ & $\begin{array}{l}\text { Basado en la } \\
\text { experimenta } \\
\text { ción }\end{array}$ \\
\hline
\end{tabular}


Aumentamos el universo (1으, 2을 으)

\begin{tabular}{|c|c|c|c|c|c|}
\hline $\begin{array}{l}\text { Los planetas, } \\
\text { El sol, } \\
\text { Sistema } \\
\text { Solar. Día y } \\
\text { noche }\end{array}$ & $\begin{array}{l}\text { CCPC } \\
\text { ERHC }\end{array}$ & $\begin{array}{l}\text { N3. } \\
\text { Utilización } \\
\text { del } \\
\text { programa } \\
\text { Aurasma }\end{array}$ & $\begin{array}{l}\text { Mediador de } \\
\text { experiencias } \\
\text { con RA }\end{array}$ & $\begin{array}{l}\text { Creador de } \\
\text { recursos RA }\end{array}$ & $\begin{array}{l}\text { Basado en } \\
\text { proyectos }\end{array}$ \\
\hline
\end{tabular}

Descubrimos los instrumentos musicales (3으)

\begin{tabular}{|c|c|c|c|c|c|}
\hline $\begin{array}{l}\text { Sonoridad y } \\
\text { ondas }\end{array}$ & $\begin{array}{l}\text { CCPC } \\
\text { ERHC }\end{array}$ & $\begin{array}{l}\text { N3. } \\
\text { Utilización } \\
\text { del } \\
\text { programa } \\
\text { Aurasma }\end{array}$ & $\begin{array}{l}\text { Facilitador } \\
\text { aprendizaje } \\
\text { con RA }\end{array}$ & $\begin{array}{l}\text { Explorador } \\
\text { de recursos } \\
\text { RA }\end{array}$ & $\begin{array}{l}\text { Basado en la } \\
\text { experimenta } \\
\text { ción }\end{array}$ \\
\hline
\end{tabular}

Animales

\begin{tabular}{llllll}
\hline Animales & ERHC & N2: & Facilitador & Explorador & Basado en \\
& APGPC & Utilización & aprendizaje & de recursos & proyectos \\
& de & con RA & RA & \\
& marcadores & & \\
& & &
\end{tabular}

Tabla 1. Experiencias formativas con RA desarrolladas en Educación Infantil

El profesorado de Educación Infantil efectuó diversas prácticas formativas con RA, tales como Aumentamos el universo. En ella, los alumnos de los tres cursos de Infantil elaboran un lapbook que contiene objetos en RA para abordar contenidos específicos sobre los planetas, el sol, el sistema solar, la luna y entender las diferencias existentes entre el día y la noche. En esta actividad, los estudiantes participaron activamente creando contenidos aumentados con la ayuda y asesoramiento del profesorado, desarrollando las competencias de comprensión de conceptos o procesos científicos y explicación de la realidad y hechos científicos. Por su parte, en Identificamos espacios con $Q R$, se proponían a los escolares de 3으 curso pequeños recorridos por el centro, activando entre otros aspectos su orientación espacial y el reconocimiento de las diferencias entre norte, sur, este y oeste, al tiempo que descubrían diversos lugares del colegio, impulsando la competencia de comprensión de conceptos o procesos científicos. Con esta propuesta, los alumnos experimentaban la orientación espacial mediante el escaneo y exploración de los códigos QR, al ofrecerles pistas para que se dirigiesen hacia donde se les indicaba iniciando el recorrido planteado. Por último, otra de las experiencias realizadas en infantil se basó en colorear láminas que sirvieron como marcadores, con objeto de adquirir conocimientos sobre los animales.

\subsection{Educación Primaria}

Se primó la enseñanza de contenidos y conceptos relacionados con la ciencia apelando al uso de aplicaciones y recursos de RA, aprovechando su condición lúdica para hacer posible la aproximación a contenidos y conceptos abstractos y reducir su complejidad cognitiva. Así, en 
este nivel educativo se emplearon recursos innovadores basados en la utilización de la RA como una tecnología disruptiva para captar la atención del alumnado, al ofrecerles grandes oportunidades para acercarse los contenidos científicos:

\begin{tabular}{|c|c|c|c|c|c|}
\hline $\begin{array}{l}\text { Contenidos } \\
\text { científicos }\end{array}$ & $\begin{array}{l}\text { Competencias } \\
\text { científicas }\end{array}$ & $\begin{array}{l}\text { Nivel de } \\
\text { RA }\end{array}$ & Rol docente & Rol alumnos & $\begin{array}{l}\text { Metodologí } \\
\text { a }\end{array}$ \\
\hline \multicolumn{6}{|c|}{ Árboles y arbustos del colegio (4으) } \\
\hline $\begin{array}{l}\text { Flora del } \\
\text { entorno } \\
\text { inmediato del } \\
\text { colegio }\end{array}$ & $\begin{array}{l}\text { CCPC } \\
\text { ERHC }\end{array}$ & $\begin{array}{l}\text { N1. } \\
\text { Mediante } \\
\text { códigos } \\
\text { QR }\end{array}$ & $\begin{array}{l}\text { Facilitador } \\
\text { aprendizaje } \\
\text { con RA }\end{array}$ & $\begin{array}{l}\text { Explorador } \\
\text { de recursos } \\
\text { RA }\end{array}$ & $\begin{array}{l}\text { Basado en } \\
\text { proyectos }\end{array}$ \\
\hline
\end{tabular}

Water lapbook (1으, 2ㅇ, 3ㅇ, 4으, 5으 y 6으)

\begin{tabular}{|c|c|c|c|c|c|}
\hline $\begin{array}{l}\text { Ciclo del agua, } \\
\text { flotabilidad, } \\
\text { consumo } \\
\text { responsable. }\end{array}$ & $\begin{array}{l}\text { CCPC } \\
\text { ERHC }\end{array}$ & $\begin{array}{l}\text { N3. } \\
\text { Utilización } \\
\text { del } \\
\text { programa } \\
\text { Aurasma y } \\
\text { Quiver-ed }\end{array}$ & $\begin{array}{l}\text { Mediador } \\
\text { de } \\
\text { experiencia } \\
\text { s con RA }\end{array}$ & $\begin{array}{l}\text { Creador de } \\
\text { recursos RA }\end{array}$ & $\begin{array}{l}\text { Basado en } \\
\text { proyectos }\end{array}$ \\
\hline
\end{tabular}

Animalandia (1으 y $2 \stackrel{\circ}{)}$

\begin{tabular}{|c|c|c|c|c|c|}
\hline $\begin{array}{l}\text { Animales } \\
\text { vertebrados }\end{array}$ & СCPC & $\begin{array}{l}\text { N1. } \\
\text { Mediante } \\
\text { códigos } \\
\text { QR }\end{array}$ & $\begin{array}{l}\text { Proveedor } \\
\text { de recursos } \\
\text { de RA }\end{array}$ & $\begin{array}{l}\text { Consumidor } \\
\text { de recursos } \\
\text { RA }\end{array}$ & $\begin{array}{l}\text { Apoyo } \\
\text { metodologí } \\
\text { a } \\
\text { tradicional }\end{array}$ \\
\hline
\end{tabular}

How to make butter (2ㅇ)

\begin{tabular}{llllll}
\hline Procedimiento & CCPC & N1. & Facilitador & Explorador & Basado en \\
s químicos en & ERHC & Mediante & $\begin{array}{l}\text { aprendizaje } \\
\text { de recursos }\end{array}$ & proyectos \\
la elaboración & APGPC & códigos & con RA & RA & \\
de la & & QR & & & \\
mantequilla & & & & & \\
\hline
\end{tabular}

\section{Ciclo del agua (3으}

\begin{tabular}{|c|c|c|c|c|c|}
\hline $\begin{array}{l}\text { Fases y } \\
\text { proceso del } \\
\text { ciclo del agua }\end{array}$ & $\begin{array}{l}\text { CCPC } \\
\text { ERHC }\end{array}$ & $\begin{array}{l}\text { N1. } \\
\text { Mediante } \\
\text { códigos } \\
\text { QR }\end{array}$ & $\begin{array}{l}\text { Proveedor } \\
\text { de recursos } \\
\text { de RA }\end{array}$ & $\begin{array}{l}\text { Consumidor } \\
\text { de recursos } \\
\text { RA }\end{array}$ & $\begin{array}{l}\text { Apoyo } \\
\text { metodologí } \\
\text { a } \\
\text { tradicional }\end{array}$ \\
\hline
\end{tabular}


Magnets and materials (3ㅇ)

\begin{tabular}{|c|c|c|c|c|c|}
\hline $\begin{array}{l}\text { Magnetismos: } \\
\text { explicación, } \\
\text { dinámica y } \\
\text { funcionamient } \\
\text { o }\end{array}$ & $\begin{array}{l}\text { CCPC } \\
\text { ERHC } \\
\text { APGPC }\end{array}$ & $\begin{array}{l}\text { N1. } \\
\text { Mediante } \\
\text { códigos } \\
\text { QR }\end{array}$ & $\begin{array}{l}\text { Facilitador } \\
\text { aprendizaje } \\
\text { con RA }\end{array}$ & $\begin{array}{l}\text { Explorador } \\
\text { de recursos } \\
\text { RA }\end{array}$ & $\begin{array}{l}\text { Basado en } \\
\text { proyectos y } \\
\text { experiment } \\
\text { ación }\end{array}$ \\
\hline
\end{tabular}

Plants (5)

\begin{tabular}{|c|c|c|c|c|c|}
\hline $\begin{array}{l}\text { Flora de la } \\
\text { región }\end{array}$ & $\begin{array}{l}\text { CCPC } \\
\text { ERHC }\end{array}$ & $\begin{array}{l}\text { N1. } \\
\text { Mediante } \\
\text { códigos } \\
\text { QR }\end{array}$ & $\begin{array}{l}\text { Proveedor } \\
\text { de recursos } \\
\text { de RA }\end{array}$ & $\begin{array}{l}\text { Consumidor } \\
\text { de recursos } \\
\text { RA }\end{array}$ & $\begin{array}{l}\text { Apoyo } \\
\text { metodologí } \\
\text { a } \\
\text { tradicional }\end{array}$ \\
\hline \multicolumn{6}{|c|}{ Safer Internet Day (5으) } \\
\hline $\begin{array}{l}\text { Bases y } \\
\text { funcionamie } \\
\text { nto de redes } \\
\text { de } \\
\text { comunicació } \\
\text { n }\end{array}$ & $\begin{array}{l}\text { CCPC } \\
\text { ERHC }\end{array}$ & $\begin{array}{l}\text { N1.código } \\
\text { SQR } \\
\text { N3: } \\
\text { Aurasma }\end{array}$ & $\begin{array}{l}\text { Facilitador } \\
\text { aprendizaje } \\
\text { con RA }\end{array}$ & $\begin{array}{l}\text { Explorador } \\
\text { de recursos } \\
\text { RA }\end{array}$ & $\begin{array}{l}\text { Basado en } \\
\text { proyectos }\end{array}$ \\
\hline
\end{tabular}

Machines around us (5ㅇ)

\begin{tabular}{|c|c|c|c|c|c|}
\hline $\begin{array}{l}\text { Identificació } \\
\text { n de } \\
\text { máquinas del } \\
\text { centro }\end{array}$ & $\begin{array}{l}\text { CCPC } \\
\text { ERHC } \\
\text { APGPC }\end{array}$ & $\begin{array}{l}\text { N1. } \\
\text { Mediante } \\
\text { códigos } \\
\text { QR }\end{array}$ & $\begin{array}{l}\text { Facilitador } \\
\text { aprendizaje } \\
\text { con RA }\end{array}$ & $\begin{array}{l}\text { Explorador } \\
\text { de recursos } \\
\text { RA }\end{array}$ & $\begin{array}{l}\text { Basado en } \\
\text { proyectos y } \\
\text { experiment } \\
\text { ación }\end{array}$ \\
\hline
\end{tabular}

Etapas de la historia (5으)

\begin{tabular}{|c|c|c|c|c|c|}
\hline $\begin{array}{l}\text { Etapas de la } \\
\text { historia: } \\
\text { Prehistoria, } \\
\text { Edad Antigua } \\
\text { y Edad } \\
\text { Media. }\end{array}$ & $\begin{array}{l}\text { CCPC } \\
\text { IDPC }\end{array}$ & $\begin{array}{l}\text { N1. } \\
\text { Mediante } \\
\text { códigos } \\
\text { QR }\end{array}$ & $\begin{array}{l}\text { Facilitador } \\
\text { aprendizaje } \\
\text { con RA }\end{array}$ & $\begin{array}{l}\text { Explorador } \\
\text { de recursos } \\
\text { RA }\end{array}$ & $\begin{array}{l}\text { Basado en } \\
\text { proyectos }\end{array}$ \\
\hline
\end{tabular}

Plantas y pájaros ... de mi pueblo (6으)

\begin{tabular}{|c|c|c|c|c|c|}
\hline $\begin{array}{l}\text { Flora y fauna } \\
\text { del entorno } \\
\text { inmediato }\end{array}$ & $\begin{array}{l}\text { CCPC } \\
\text { ERHC }\end{array}$ & $\begin{array}{l}\text { N1. } \\
\text { Mediante } \\
\text { códigos } \\
\text { QR }\end{array}$ & $\begin{array}{l}\text { Facilitador } \\
\text { aprendizaje } \\
\text { con RA }\end{array}$ & $\begin{array}{l}\text { Explorador } \\
\text { de recursos } \\
\text { RA }\end{array}$ & $\begin{array}{l}\text { Basado en } \\
\text { proyectos }\end{array}$ \\
\hline
\end{tabular}


Electricidad y magnetismo (6ㅇ)

\begin{tabular}{llllll}
\hline $\begin{array}{l}\text { Conceptos } \\
\text { básicos }\end{array}$ & CCPC & N1. & Facilitador & Explorador & Basado en \\
sobre & ERHC & $\begin{array}{l}\text { Mediante } \\
\text { códigos }\end{array}$ & $\begin{array}{l}\text { aprendizaje } \\
\text { con RA }\end{array}$ & RA recursos & proyectos \\
$\begin{array}{l}\text { Electricidady } \\
\text { magnetismo }\end{array}$ & & QR & & \\
\hline
\end{tabular}

Flora y fauna del Parque Isabel la Católica (Gijón) (5o)

\begin{tabular}{|c|c|c|c|c|c|}
\hline $\begin{array}{l}\text { Flora y fauna } \\
\text { del entorno } \\
\text { inmediato }\end{array}$ & $\begin{array}{l}\text { CCPC } \\
\text { ERHC }\end{array}$ & $\begin{array}{l}\text { N1. } \\
\text { Mediante } \\
\text { códigos } \\
\text { QR }\end{array}$ & $\begin{array}{l}\text { Facilitador } \\
\text { aprendizaje } \\
\text { con RA }\end{array}$ & $\begin{array}{l}\text { Creador de } \\
\text { recursos RA }\end{array}$ & $\begin{array}{l}\text { Basado en } \\
\text { proyectos }\end{array}$ \\
\hline
\end{tabular}

Tabla 2. Experiencias formativas con RA desarrolladas en Educación Primaria

En Educación Primaria se optó principalmente por la utilización de códigos QR (Tabla 2), debido a que para la mayoría del profesorado participante en el proyecto esta era su primera aproximación a la RA, y ello entrañaba menor dificultad. Así, se decantaron por el diseño de actividades formativas basadas en el nivel I, atendiendo a su grado de inmersión tecnológica. Entre ellas, se encuentra Árboles y arbustos del colegio, con la que los alumnos de 40 de primaria conocieron y exploraron la flora del entorno inmediato al colegio, elaborando diferentes itinerarios didácticos que, a modo de gymkhana, favorecían el desarrollo de las competencias de comprensión de conceptos o procesos científicos y explicación de la realidad y hechos científicos. Una experiencia semejante fue Flora y fauna del parque Isabel la Católica, en la que los participantes elaboraron fichas -en español e inglés- con información sobre las plantas y animales de un espacio próximo al colegio y generaron códigos $Q R$, que situaron sobre un mapa.

Igualmente, surgieron varias iniciativas desde las aulas bilingües, como How to make butter de 2 o de primaria, donde se abordan contenidos próximos a la química cómo la elaboración de la mantequilla; Magnets and materials de 3 o curso, en la que los alumnos abordan contenidos relacionados con el magnetismo o Machines around us de 5o curso que, a modo de gymkhana, los estudiantes debían identificar y conocer las máquinas del centro. En ellas, los alumnos debían explorar los contenidos escondidos tras los códigos QR elaborados por los docentes para facilitar la adquisición de nuevos aprendizajes.

Otra de las experiencias se enmarca, a diferencia del resto, en el campo de las ciencias sociales, dirigiéndose al aprendizaje de las distintas etapas de la historia. Concretamente, el alumnado elaboró unas líneas de tiempo agregando y seleccionando objetos representativos de cada etapa. Con estos materiales se preparó una exposición en la que se insertaron códigos QR. Finalmente, el alumnado cumplimentó un cuestionario con preguntas sobre cada periodo histórico, a partir de la información recabada a través de los códigos QR.

Sin embargo, otras iniciativas se centraron en otorgarle a los estudiantes un mayor protagonismo al participar activamente en la creación de los recursos de RA, como Water 
lapbook. En ella, el alumnado de primaria creó colaborativamente un lapbook con recursos aumentados, empleando los programas Aurasma y Quiver-ed, para profundizar en el conocimiento de las fases del ciclo del agua, la importancia de su consumo responsable, la flotabilidad, etc., trabajando las competencias de comprensión de conceptos o procesos científicos y explicación de la realidad y hechos científicos.

Por otro lado, cabe destacar que gran parte de las actividades desarrolladas en esta etapa adoptaron la modalidad de proyectos. En ellos, los estudiantes exploran los nuevos contenidos apoyados en RA, de manera lúdica e interactiva, para adquirir nuevos conocimientos y habilidades. Así, en Electricidad y magnetismo de 6으 curso, los alumnos se aproximan y exploran las características y posibilidades que estos elementos ofrecen usando códigos QR, para posteriormente elaborar diferentes trabajos multimedia donde se detallen sus posibles aplicaciones y usos de un modo creativo e innovador.

\subsection{Educación Secundaria y Bachillerato}

La enseñanza de los contenidos científicos -tal como se contempla en el currículum oficial-, se concreta de forma distinta en cada nivel educativo dada su complejidad. Generalmente, se aborda con el apoyo de actividades basadas en la observación y la experimentación, donde la condición lúdica de la RA juega un papel importante para favorecer el aprendizaje de hechos y procesos desarrollados en lapsos de tiempo prolongado.

\begin{tabular}{lllll}
\hline $\begin{array}{l}\text { Contenidos } \\
\text { científicos }\end{array}$ & $\begin{array}{l}\text { Competencia } \\
\text { s científicas }\end{array}$ & Nivel de RA & Rol docente & Rol alumnos Metodología \\
\hline
\end{tabular}

Mujeres en la ciencia (1ㅇ, 2으, 3으, 4ㅇ y Bachillerato)

\begin{tabular}{|c|c|c|c|c|c|}
\hline $\begin{array}{l}\text { Biografía y } \\
\text { obra de } \\
\text { mujeres } \\
\text { científicas }\end{array}$ & $\begin{array}{l}\text { IDPC } \\
\text { APGPC }\end{array}$ & $\begin{array}{l}\text { N1. } \\
\text { Mediante } \\
\text { códigos QR }\end{array}$ & $\begin{array}{l}\text { Mediador de } \\
\text { Experiencias } \\
\text { con RA }\end{array}$ & $\begin{array}{l}\text { Creador de } \\
\text { Recursos RA }\end{array}$ & $\begin{array}{l}\text { Basado en } \\
\text { proyectos }\end{array}$ \\
\hline
\end{tabular}

Un paseo por la antigua Grecia (10 ESO)

\begin{tabular}{|c|c|c|c|c|c|}
\hline $\begin{array}{l}\text { Arquitectura } \\
\text { urbanismo y } \\
\text { creencias en } \\
\text { la antigua } \\
\text { Grecia }\end{array}$ & СCPC & $\begin{array}{l}\text { N1. } \\
\text { Mediante } \\
\text { códigos QR }\end{array}$ & $\begin{array}{l}\text { Mediador de } \\
\text { Experiencias } \\
\text { con RA }\end{array}$ & $\begin{array}{l}\text { Explorador } \\
\text { de recursos } \\
\text { RA }\end{array}$ & $\begin{array}{l}\text { Basado en } \\
\text { Proyectos }\end{array}$ \\
\hline
\end{tabular}

Biología y geografía de Asturias 1으 y 2을 ESO

\begin{tabular}{|c|c|c|c|c|c|}
\hline $\begin{array}{l}\text { Biología: } \\
\text { invertebrado } \\
\text { s. La avispa } \\
\text { asiática. }\end{array}$ & $\mathrm{ERHC}$ & $\begin{array}{l}\text { N1. } \\
\text { Mediante } \\
\text { códigos QR } \\
\text { y N3: }\end{array}$ & $\begin{array}{l}\text { Mediador de } \\
\text { Experiencias } \\
\text { con RA }\end{array}$ & $\begin{array}{l}\text { Creador de } \\
\text { Recursos RA }\end{array}$ & $\begin{array}{l}\text { Basado en } \\
\text { problemas }\end{array}$ \\
\hline
\end{tabular}




\begin{tabular}{|c|c|c|c|c|c|}
\hline \multicolumn{2}{|c|}{$\begin{array}{l}\text { Geografía de } \\
\text { Asturias: } \\
\text { Municipios }\end{array}$} & \multicolumn{4}{|l|}{$\begin{array}{l}\text { utilización } \\
\text { de } \\
\text { imágenes y } \\
\text { como } \\
\text { activadores }\end{array}$} \\
\hline \multicolumn{6}{|c|}{ Reciclaje (4으) } \\
\hline $\begin{array}{l}\text { Reciclaje: } \\
\text { proceso y } \\
\text { beneficios }\end{array}$ & $\begin{array}{l}\text { ERHC } \\
\text { DEPC } \\
\text { APGPC }\end{array}$ & $\begin{array}{l}\text { N3. } \\
\text { Geolocaliza } \\
\text { ción } \\
\text { mediante } \\
\text { Eduloc }\end{array}$ & $\begin{array}{l}\text { Mediador de } \\
\text { Experiencias } \\
\text { con RA }\end{array}$ & $\begin{array}{l}\text { Creador de } \\
\text { Recursos RA }\end{array}$ & $\begin{array}{l}\text { Basado en } \\
\text { proyectos y } \\
\text { experimenta } \\
\text { ción }\end{array}$ \\
\hline
\end{tabular}

Tabla 3. Experiencias formativas con RA desarrolladas en Educación Secundaria y Bachillerato

Así, en Mujeres en la ciencia, se expusieron 25 paneles con la biografía y obra de algunas mujeres científicas (Tabla 3). Los paneles explicaban brevemente los aspectos más relevantes de su biografía y los trabajos y estudios realizados a lo largo de su carrera. La exposición se completó con animaciones, vídeos, etc. recogidos a través de códigos QR elaborados por los estudiantes para ofrecer una información complementaria a los visitantes que acudieran a la exposición. Para ello, los discentes siguieron un proceso de búsqueda, selección y tratamiento de la información que permitió recoger datos relevantes y pertinentes para incluirlos en los códigos QR.

De igual modo, en la iniciativa Reciclaje, los estudiantes de 4을 de secundaria elaboraron, utilizando la herramienta Eduloc, una guía de localización para las islas de reciclaje en un localidad asturiana incorporando explicaciones multiformato (pdf, vídeo, animación...) relacionadas con los materiales reciclables (cubos, efecto sobre fauna, flora...), estableciendo un juego de preguntas para vincular la localización de los cubos con zonas de interés de la localidad donde se ubica el centro.

En otra experiencia, el alumnado elaboró códigos QR para acceder a información elaborada en clase de Biología. Posteriormente, se creó un mapa de Asturias, añadiendo información sobre cada concejo que se activaba al escanear su imagen.

En el caso de la experiencia Un paseo por la antigua Grecia, la RA se usó en el contexto de las ciencias sociales. Los estudiantes confeccionaron un mural tomando como eje temático la arquitectura en la antigua Grecia y generaron códigos QR para ampliar la información.

En definitiva, desde los diferentes centros educativos se desarrollaron actividades formativas basadas en la utilización de la RA como recurso para potenciar la motivación e implicación de los estudiantes. Sin embargo, aunque el manejo de los recursos de RA es sencillo, se considera necesario que el profesorado posea ciertas habilidades, no solo tecnológicas sino también didáctico-metodológicas, que favorezcan su integración en las aulas de Educación Infantil, Primaria, Secundaria y Bachillerato, generando espacios donde se prime el aprendizaje por descubrimiento y la experimentación. 


\section{CONCLUSIONES}

El proyecto ACRA permitió el diseño y puesta en práctica de diversas experiencias innovadoras basadas en la utilización de la RA como recurso pedagógico en enseñanzas no universitarias. Además, ha favorecido la innovación en el ámbito escolar, promoviendo la introducción de una tecnología emergente en las aulas de Educación Infantil, Primaria, Secundaria y Bachillerato, cuya explotación didáctica es todavía relativamente escasa. Al respecto, cabe reseñar que, si bien algunos de los docentes participantes ya estaban familiarizados con el uso educativo de la RA y la habían empleado anteriormente con su alumnado, para otros era una herramienta novedosa, cuyas posibilidades no habían explorado previamente. Asimismo, las experiencias pedagógicas desarrolladas ofrecen ejemplos de buenas prácticas con RA, que ayudan a comprender mejor las potencialidades de estas tecnologías e ilustran sus aplicaciones en el aula, en el contexto de la enseñanza de las ciencias.

Las 21 experiencias presentadas ofrecen, en primer lugar, la posibilidad de adaptar los recursos y actividades con RA a diferentes etapas educativas y, por consiguiente, a diversas edades y capacidades cognitivas. Concretamente, el proyecto ACRA impulsó experiencias desde el segundo ciclo de Educación Infantil hasta Bachillerato, siendo mayoría el número de experiencias llevadas a cabo en Educación Primaria. La mayoría de las propuestas abordaron contenidos temáticos del área de las Ciencias Naturales -como la física, botánica, zoología, etc.-, revelando su adecuación para activar diversas competencias científicas. Sin embargo, algunos docentes optaron por enfocar sus experiencias al campo de las Ciencias Sociales, utilizando la RA para impulsar el aprendizaje de conocimientos de geografía o historia. Por otra parte, hay que destacar el carácter interdisciplinar de algunos de las propuestas desarrolladas donde, partiendo de un tema de ciencias como eje central, se hacía converger a otras materias, como lengua castellana, inglés, educación artística, etc.

Las competencias científicas más potenciadas fueron la Comprensión de Conceptos o Procesos Científicos y la Explicación de la Realidad o Hechos Científicos, aunque la totalidad de las experiencias ofrece ejemplos para impulsar todas las competencias científicas señaladas.

En algunos casos, la actividad diseñada requirió la exploración del entorno como punto de partida, mostrando la compatibilidad del trabajo de campo con el uso de las tecnologías de RA. Otras veces, la tarea implicó una fase inicial de documentación sobre el tema, ejercitando las habilidades de búsqueda, selección y procesamiento de la información, para la elaboración posterior de contenidos aumentados.

Las experiencias constituyen un repertorio variado para integrar la RA en el aula. En cuanto al tipo de $R A$ empleado, la mayoría de los docentes optó por elegir recursos del nivel 1 (códigos QR), pero también hay ejemplos de los niveles 2 (marcadores) y 3 (imágenes y objetos como activadores). En ningún caso se utilizaron procedimientos del nivel 4, probablemente por la carencia de soportes tecnológicos necesarios. En cuanto al rol del docente, algunos actuaron básicamente como proveedores de recursos para utilizar en sus clases, mientras que otros adoptaron el papel de facilitador del aprendizaje con RA o de mediador de experiencias con RA -en los niveles superiores-. Por otra parte, si bien en 
algunos casos el alumnado se limitó a explorar recursos RA para familiarizarse con ellos, o fueron meros consumidores de recursos diseñados por otros, en muchas experiencias participaron activamente en el diseño y elaboración de sus propios recursos. Como es lógico, este rol más activo del alumnado predominó en las etapas superiores, aunque se dio también en al menos una experiencia de Infantil.

Por otra parte, aunque en algún caso la RA se concibió como instrumento de apoyo en el marco de una metodología tradicional, predominaron metodologías más activas, optándose mayoritariamente por el aprendizaje basado en proyectos, $y$, en menor medida, por la experimentación y el aprendizaje basado en problemas. Por lo general, el alumnado trabajó de forma cooperativa e igualmente se propició la cooperación entre docentes de diferentes cursos y áreas.

En definitiva, el análisis de las experiencias derivadas del proyecto ACRA pone de manifiesto la versatilidad de la RA en el contexto educativo, adaptable a diferentes etapas y niveles, dado que ha permitido diseñar actividades con niveles variados de dificultad. Asimismo, se revela como un recurso eficaz para potenciar diferentes aprendizajes $y$, en especial, los de carácter científico. En este campo, cabe señalar también la posibilidad de combinar las actividades apoyadas en RA tanto con el trabajo de campo como con las tareas de búsqueda y procesamiento de información. De igual modo, las actividades apoyadas en estas tecnologías parecen adaptarse bien a las metodologías que potencian el rol activo del alumno, como el aprendizaje por proyectos, y resultan eficaces para promover el trabajo colaborativo. Por lo que se puede afirmar que el alumnado participante en el proyecto ACRA ha ejercitado sus competencias científicas, al tiempo que se favorecía su competencia digital y sus habilidades para el trabajo en equipo.

\section{REFERENCIAS BIBLIOGRÁFICAS}

Binkley, M., Erstad, O., Herman, J., Raizen, S., Ripley, M., \& Rumble, M. (2010). Defining 21st century skills. Disponible en: https://doi.org/10.1007/978-94-007-2324-5 2

Bronack, S.C. (2011). The role of immersive media in online education. Journal of Continuing Higher Education, 59(2), 113-117.

Cabero, J. (2010). Los retos de la integración de las TICs en los procesos educativos. Límites y posibilidades. Perspectiva Educacional, Formación de Profesores, 49(1), 32-61.

Caro, J.L., Luque, A.M., \& Zayas, B. (2014). Aplicaciones tecnológicas para la promoción de los recursos turísticos culturales. XVI Congreso Nacional de Tecnologías de la Información Geográfica, 25-27, Junio 2014. Alicante: Universidad de Alicante. Disponible en https://riuma.uma.es/xmlui/handle/10630/7889

Confederación de Sociedades Científicas de España, COSCE (2011). Informe ENCIENDE: Enseñanza de las ciencias para la didáctica escolar para edades tempranas en España. Madrid: Rubes. 
Cañal, P. (2012). ¿Cómo evaluar la competencia científica?. Investigación en la Escuela, 78, 515.

Cawood S. \& Fiala M. (2008). Augmented Reality: A Practical Guide. Denver: Pragmatic Bookshelf.

Cubillo, J., Martín-Gutiérrez, S., Castro, M., \& Colmenar, A. (2014). Recursos digitales autónomos mediante realidad aumentada. RIED. Revista Iberoamericana de Educación a Distancia, 17(2), 241-274.

Davis, E.A. \& Krajcik, J.S.(2005). Designing educative curriculum materials to promote teacher learning. Educational Researcher, 34(3), 3-14.

De la Torre, J., Martín-Dorta, N., Saorín, J. L., Carbonell, C. \& Contero, M. (2015). Entorno de aprendizaje ubicuo con realidad aumentada y tabletas para estimular la comprensión del espacio tridimensional. Revista de Educación a Distancia, 37. Disponible en http://revistas.um.es/red/article/view/234041

De Pedro, J., \& Martínez-Méndez, C.L. (2012). Realidad Aumentada: Una Alternativa Metodológica en la Educación Primaria Nicaragüense. IEEE-RITA, 7(2), 102-108.

Del Cerro, F. \& Morales, G. (2017). Realidad Aumentada como herramienta de mejora de la inteligencia espacial en estudiantes de educación secundaria. Revista de Educación a Distancia, 54. Disponible en http://revistas.um.es/red/article/view/298831

Del Moral, M.E. y Villalustre, L. (2013). Realidad aumentada: experimentando en el aula en 3D. En R. Ron, A. Álvarez \& P. Núñez (Coords.). Smartphones y tablets: ¿enseñan o distraen? (pp. 107-124). Madrid: ESIC Editorial

Hung, Y.H., Chen, C.H., \& Huang, S.W. (2017) Applying augmented reality to enhance learning: a study of different teaching materials. Journal of Computer Assisted Learning, 33, 252-266.

Leiva, J.J., \& Moreno, N.M. (2015). Tecnologías de geolocalización y realidad aumentada en contextos educativos: experiencias y herramientas didácticas. DIM: Didáctica, Innovación y Multimedia, 31, 1-18.

Martí Feixas, J. (2012). Aprender ciencias en la educación primaria. Barcelona: Graó.

Méndez, P.J. (2012). Mundos Cambiantes: La Tecnología y la Educación 3.0. Revista Complutense de Educación, 23(1), 11-22.

National Research Council, NRC (2007). Taking science to school: learning and teaching science in grades $\mathrm{K}-8$. Washington, DC: National Academic Press. 
Roblizo, M., Sánchez-Pérez, M., \& Cózar, R. (2015). El reto de la competencia digital en los futuros docentes de Infantil, Primaria y Secundaria: los estudiantes de grado y máster de Educación ante las TIC. Prisma Social, 15, 254-295.

Rocard, M., Csermely, P., Jorde, D., Lenzen, D., Walwerg-Henriksson, H. \& Hemmo, V. (2007). Science Education Now: A Renewed Pedagogy for the Future of Europe. Bruselas. Disponible en:

http://ec.europa.eu/research/science-society/document library/pdf 06/report-rocard-onscience-education en.pdf

Rosado, J.J. (2010). Futuro de la realidad aumentada. En blog: Mi cuarta dimensión: desfragmentado el Universo, Tecnologías y otros sentimientos. Recuperado de http://coppernic.blogspot.com.es/2010/09/futuro-de-la-realidad-aumentada.html

Salinas, J. (2012). La investigación ante los desafíos de los escenarios de aprendizaje futuros. RED. Revista de Educación a Distancia, 32. Disponible en http://www.um.es/ead/red/32/salinas.pdf

Sánchez-Prieto, J.C., Olmos, S., García-Peñalvo, F. J., \& Torrecilla, E.M. (2016). Las tabletas digitales en educación formal: características principales y posibilidades pedagógicas. En A.I. Callejas, J.V. Salido, Ó. Jerez García (coords.) (2016). Competencia digital y tratamiento de la información: Aprender en el siglo XXI. (pp. 153, 269). Cuenca: Universidad de Castilla-La Mancha.

Sancho, J.M., Correa, J.M., Giró, X., \& Fraga, L. (2014). Aprender a ser docente en un mundo en cambio. In Simposio Internacional (pp. 290-294). Barcelona: Dipòsit Digital de la Universitat de Barcelona.

Solano, C.A., Díaz, Casas, J.F., \& Guevara, J.C. (2015). Aplicación móvil de realidad aumentada para la enseñanza de la clasificación de los seres vivos a niños de tercer grado. Ingeniería, 20(1), 79-93. DOI: https://doi.org/10.14483/udistrital.jour.reving.2015.1.a05

Toledo, P. \& Sánchez-García, J.M. (2017). Realidad Aumentada en Educación Primaria: efectos sobre el aprendizaje. Revista Latinoamericana de Tecnología EducativaRELATEC, 16(1), 79-92. DOI: https://doi.org/10.17398/1695-288X.16.1.79

Villalustre, L. \& Del Moral, M.E. (Coords.) (2016a). Experiencias interactivas con realidad aumentada en las aulas. Barcelona: Octaedro.

Villalustre, L. \& Del Moral, M.E. (2016b). Itinerarios interactivos con geolocalización y realidad aumentada para un aprendizaje ubicuo en la formación inicial de docentes de educación infantil. En L. Villalustre y M.E. Del Moral (coords.) (2016). Experiencias interactivas con realidad aumentada en las aulas (pp. 31-53). Barcelona: Octaedro. 
Villalustre, L. \& Del Moral, M.E. (2017). Juegos perceptivos con realidad aumentada para trabajar contenido científico. Revista Educação, Formação \& Tecnologias, 10(1), 3646.

Villalustre, L., Del Moral, M.E., Neira-Piñeiro, M.R. y Herrero, M. (2017). Proyecto ACRA: Aprender Ciencias con Realidad Aumentada. XX Congreso Internacional Edutec'2017: Investigación, innovación y tecnologías, la triada para transformar los procesos formativos. 8-10 de noviembre. Santiago de Chile: Universidad Santiago de Chile.

\section{Para referenciar este artículo:}

Lourdes-Villalustre, M., Neira-Piñero, Maㅡ R. \& Herrero-Vázquez, M. (2017) En ciencias con realidad aumentada en los niveles pre-universitarios. EDUTEC, Revista electrónica de Tecnología Educativa, 62. Recuperado de: http://dx.doi.org/10.21556/edutec.2017.62.1009 